\title{
Evaluation of beta-blockers on left ventricular dyssynchrony and reverse remodeling in idiopathic dilated cardiomyopathy: A randomized trial of carvedilol and metoprolol
}

\author{
Mehmet Gungor Kaya ${ }^{1}$, Bahadir Sarli ${ }^{1}$, Mahmut Akpek ${ }^{1}$, Esma Gunduz Kaya ${ }^{2}$, \\ Mikail Yarlioglues ${ }^{1}$, Ramazan Topsakal ${ }^{1}$, Yat-Yin Lam $^{3}$ \\ ${ }^{1}$ Department of Cardiology, Erciyes University School of Medicine, Kayseri, Turkey \\ ${ }^{2}$ Department of Microbiology, Erciyes University School of Medicine, Kayseri, Turkey \\ ${ }^{3}$ Department of Medicine and Therapeutics, Prince of Wales Hospital, \\ The Chinese University of Hong Kong, Hong Kong
}

\begin{abstract}
Background: The effect of $\beta$-blockage on cardiac dyssynchrony in idiopathic dilated cardiomyopathy $(I D C)$ is unknown. This study evaluated the impact of carvedilol and metoprolol succinate on left ventricular (LV) dyssynchrony and reverse remodeling in IDC.

Methods: In this small, prospective, double-blind study, we randomly assigned 81 IDC patients to receive carvedilol or metoprolol succinate. Echocardiographic measurements (dyssynchrony, $L V$ volumes and ejection fraction [EF]) and $N$-terminal pro- $B$-type natriuretic peptide (NT-proBNP) levels were obtained at baseline and at first and sixth month of therapy.

Results: A total of 74 (91\%) patients completed all investigations at sixth month (38 and 36 taking carvedilol and metoprolol succinate, respectively). In the carvedilol group, reduction in $L V$ end diastolic volume ( $\triangle L V E D V$ at 6 months, $50 \pm 15 \mathrm{~mL}$ to $40 \pm 17 \mathrm{~mL}, p=0.03)$ and increase in LVEF ( $\triangle L V E F, 7 \pm 2 \%$ to $5 \pm 3 \%, p=0.02)$ was higher compared to the metoprolol group. Also improvement in inter-ventricular dyssynchrony achieved with carvedilol was higher than metoprolol ( $\Delta$ interventricular delay at 6 months, $11 \pm 8$ ms to $6 \pm 7 \mathrm{~ms}, p=0.03$ ). However, improvement in intraventricular dyssynchrony was similar in the two groups ( $\Delta$ intraventricular delay, $9 \pm 7 \mathrm{~ms}$ to $9 \pm 6 \mathrm{~ms}, p=0.91$ ). Improvements in LV mechanical dyssynchrony and reverse remodeling achieved with both drugs were accompanied by reduction in NT-proBNP levels in both carvedilol and metoprolol groups $(1614 \pm 685 \mathrm{pg} / \mathrm{mL}$ to $654 \pm$ $\pm 488 \mathrm{pg} / \mathrm{mL}$ and $1686 \pm 730 \mathrm{pg} / \mathrm{mL}$ to $583 \pm 396 \mathrm{pg} / \mathrm{mL}$, respectively, $p<0.001$ for both). Conclusions: Although reduction in LVEDV and increase in LVEF was higher with carvedilol, improvement in intraventricular dyssynchrony was similar in carvedilol and metoprolol groups. (Cardiol J 2014; 21, 4: 434-441)
\end{abstract}

Key words: carvedilol, metoprolol succinate, intraventricular dyssynchrony, idiopathic dilated cardiomyopathy

Address for correspondence: Associate Professor Yat-Yin Lam, Institute of Vascular Medicine and Division of Cardiology, Department of Medicine and Therapeutics, Prince of Wales Hospital and Li Ka Shing Institute of Health and Sciences, The Chinese University of Hong Kong, Hong Kong, tel: 852-2632 1299, fax: 852-2637 3852, e-mail: yylam@cuhk.edu.hk Received: 03.07.2013

Accepted: 16.09.2013 


\section{Introduction}

Heart failure (HF) is associated with marked morbidity, mortality and frequent hospitalizations in patients over 65 years of age. Treatment of HF in order to reduce its morbidity and mortality still remains one of the major challenges in health care practice. In approximately $30 \%$ of systolic $\mathrm{HF}$ patients, the disease affects the conduction system and leads to delayed electrical activation of the lateral part of the left ventricle (LV) [1]. Such intraventricular conduction delay, conventionally defined as electrocardiographic (ECG) QRS complex duration $>120 \mathrm{~ms}$, is known to be associated with adverse LV remodeling, clinical worsening and increased mortality [2]. Recent studies showed that various novel echocardiographic indices were more reliable than QRS duration in revealing underlying mechanical dyssynchrony and predicting clinical improvement after biventricular pacing [3].

Specific $\beta$-blockers such as carvedilol, metoprolol succinate, and bisoprolol decrease deaths and HF hospitalizations in patients with chronic systolic HF by reversing LV dilation and improving cardiac performance [4-7]. However, there are limited data on whether these $\beta$-blockers can influence cardiac mechanical dyssynchrony in patients with idiopathic dilated cardiomyopathy (IDC) [8]. In this study, we aimed to investigate the effect of carvedilol and metoprolol succinate on LV intra- and interventricular dyssynchrony and reverse remodeling in IDC patients.

\section{Methods}

\section{Patient selection}

Between August 2007 and June 2008, 104 consecutive IDC patients were identified. However, only 81 patients fulfilled the inclusion criteria and were enrolled in the study. Inclusion criteria were: (1) LV ejection fraction (LVEF) $<40 \%$, (2) chronic stable HF with New York Heart Association (NYHA) functional class II or III, (3) no significant coronary artery stenosis confirmed by coronary angiography, (4) sinus rhythm, (5) no prior administration of $\beta$-blocker therapy, and (6) having mechanical intraventricular dyssynchrony as assessed by echocardiography. Patients with prior history of coronary artery disease, cardiac surgery, significant valvular heart disease, pacemaker implantation, and contra-indications to $\beta$-blockers were excluded. All patients received either maximal tolerated dose of ramipril with target dose of $10 \mathrm{mg} / \mathrm{d}$ or candesartan with target dose of $16 \mathrm{mg} / \mathrm{d}$ in whom angiotensin-converting enzyme inhibitors (ACEI) was not tolerated. Adequate furosemide therapy was added if needed. All medications were optimized at least 6-months before enrollment and remained unchanged during the study period.

Eighty-one patients were consecutively randomized in 1:1 ratio to receive carvedilol or metoprolol succinate. All patients signed an informed consent approved by our institutional review board and ethics committee. A total of 41 and 40 patients were assigned to receive carvedilol $3.125 \mathrm{mg}$ twice daily and metoprolol succinate $25 \mathrm{mg}$ once daily, respectively. The $\beta$-blocker dosage was titrated every 2 weeks to achieve target dose of $25 \mathrm{mg}$ twice daily for carvedilol and $200 \mathrm{mg}$ once daily for metoprolol succinate. Medication titration process was stopped if the patient experienced symptomatic hypotension or bradycardia.

\section{Echocardiography}

All enrolled patients received echocardiography before and at first and sixth month after randomization. They were imaged in the left lateral decubitus position using the same ultrasound system (VIVID 7, GE Vingmed Ultrasound, Horten, Norway) by 2 experienced cardiologists who were blinded to the clinical data. Two-dimensional and M-mode echocardiograms were obtained, according to the American Society of Echocardiography guidelines. The LV volumes (end-systolic and end-diastolic: LVESV and LVEDV) and LVEF were calculated from conventional apical 2- and 4-chamber images using biplane Simpson's technique [9]. $\mathrm{LV}$ reverse remodeling was defined as a reduction of LVESV $>10 \%$ at 6 -month follow-up.

Pulsed-wave tissue Doppler readings were measured from apical 4- and 5-chamber images. Gain and filter settings were adjusted to eliminate background noise for a clear tissue signal. The tissue Doppler imaging (TDI) signals were recorded at a sweep speed of $100 \mathrm{~mm} / \mathrm{s}$. The sample volume was placed in the basal portions of the septal, posterior and lateral walls of $\mathrm{LV}$ and that of right ventricular (RV) free wall. The time interval between the onset of the QRS complex and the onset of the peak systolic velocity was derived at each region. Intraventricular dyssynchrony was defined as the maximal time difference between $2 \mathrm{LV}$ walls $>60 \mathrm{~ms}$. Interventricular mechanical dyssynchrony referred to the maximal time difference between RV and LV walls > $56 \mathrm{~ms}$ [10]. All measurements were corrected for the heart rate (HR) using Bazett's formula to avoid the confounding effect of HR changes during $\beta$-blocker therapy [11]. 


\section{Biochemical analysis}

$\mathrm{N}$-terminal pro-B-type natriuretic peptide (NT-proBNP) levels were measured at baseline and 6 -months. Blood samples were collected using antecubital venous puncture and transferred to EDTA-coated test tubes. Blood samples were then centrifuged in the hospital laboratory at $4^{\circ} \mathrm{C}$ and the plasma was frozen at $-80^{\circ} \mathrm{C}$. Plasma NT-proBNP level was determined immunoenzymatically using the NT-proBNP ELISA kit (Biomedica).

\section{Reproducibility of the measurements}

Intra- and inter-observer variabilities of echocardiographic measurements were assessed in 10 randomly chosen patients. Variability was calculated as the mean percent error, derived as the difference between 2 sets of measurements, divided by the mean of the observations. Both investigators were blinded to the patients' diagnosis.

\section{Statistical analysis}

The Statistical Package for Social Sciences, version 13.0 (SPPS, Chicago, Illinois) was used for statistical analysis. Data were expressed as mean \pm standard deviation for continuous variables, and as numbers (percentages) for categorical variables. Continuous variables were analyzed by the unpaired Student's t-test. Comparisons of pre- and post-treatment variables were made using the 2-tailed, paired Student's t-test. Comparison of change in post-treatment and pre-treatment ( $\Delta=$ [post-treatment value] - [pre-treatment value]) echocardiographic parameters and NT-proBNP between the two groups was performed using Student's t-test. Pearson's or Spearman's correlation analysis was performed to estimate correlations between variables. A p value $<0.05$ was considered statistically significant.

\section{Results}

Only 74 (91\%) patients (38 in carvedilol group and 36 in metoprolol group) completed the scheduled investigations. They all received maximal tolerated doses of either carvedilol or metoprolol succinate for up to 6 months after randomization, during which time the background therapy with an angiotensin II receptor blocker/ACEI and/or diuretic was continued. Seven of 81 patients did not complete the study protocol ( 2 died, 1 developed sinus bradycardia and 4 were lost to follow-up, Fig. 1). The mean maintenance dose of $\beta$-blocker was $16 \pm$ $\pm 8 \mathrm{mg} /$ day in carvedilol group and $57 \pm 18 \mathrm{mg} /$ day in metoprolol group.

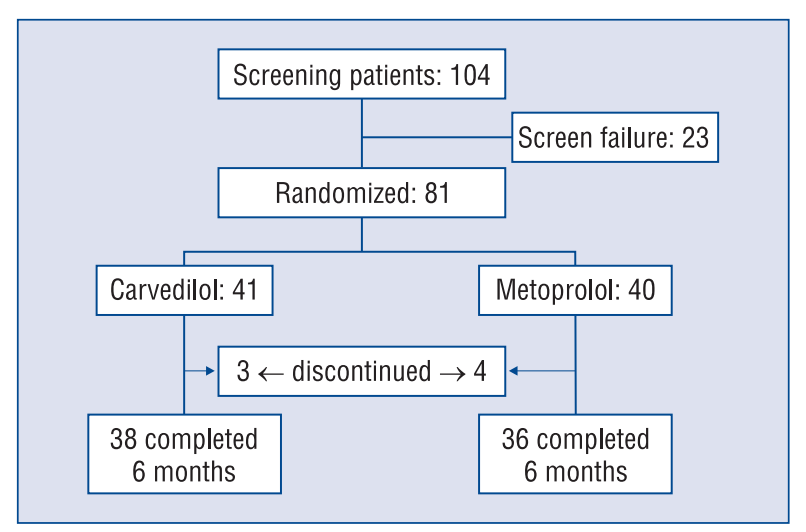

Figure 1. Disposition of the patients.

\section{Pre-treatment characteristics}

Both groups had similar age, gender and other clinical characteristics (Table 1). Baseline $\mathrm{LV}$ volumes and EF, intra- and interventricular electromechanical delay measurements, and NT-proBNP values were similar between two groups.

\section{Hemodynamic parameters}

As expected, decrease in HR was noted at 1 month in both groups (carvedilol: $81 \pm 13$ to $75 \pm 14 \mathrm{bpm}$; metoprolol: $79 \pm 15$ to $73 \pm 11 \mathrm{bpm}$, $\mathrm{p}<0.001$ for both) with no further significant change at 6 months. When compared with baseline values change in HR at 6 months were similar in the two groups $(\Delta \mathrm{HR}, 9 \pm 12$ to $9 \pm 8 \mathrm{bpm}$, $\mathrm{p}=0.91)$. Systolic blood pressure decreased from $118 \pm 16$ to $107 \pm 15 \mathrm{~mm} \mathrm{Hg}(\mathrm{p}=0.012)$ in carvedilol group and $114 \pm 14$ to $103 \pm 13 \mathrm{~mm} \mathrm{Hg}$ $(\mathrm{p}=0.035)$ in metoprolol group at 6 months. The extent of decrease in systolic blood pressure was similar between the two groups. Diastolic blood pressure significantly decreased in carvedilol (76 \pm 14 to $71 \pm 9 \mathrm{~mm} \mathrm{Hg}, \mathrm{p}=0.037$ ) but not in metoprolol group (74 \pm 12 to $70 \pm 13 \mathrm{~mm} \mathrm{Hg}$, $\mathrm{p}=0.42$ ). Both groups had similar reductions in mean NYHA functional class values at the end of the study (Table 2).

\section{$\mathrm{LV}$ reverse remodeling}

Carvedilol or metoprolol succinate therapies improved LVEF, reduced LVEDV and LVESV after 1-month treatment. Improvement in LVEF continued up to 6 months in both carvedilol and metoprolol groups $(31 \pm 6$ to $38 \pm 6 \%, \mathrm{p}<0.001$ and $32 \pm 4$ to $37 \pm 6 \%, p<0.001$, respectively). However, improvement in LVEF was higher in carvedilol group compared to metoprolol group 
Table 1. Baseline characteristics of patients with idiopathic dilated cardiomyopathy.

\begin{tabular}{lccc}
\hline & Carvedilol $(\mathbf{n}=\mathbf{3 8})$ & Metoprolol succinate $(\mathbf{n}=36)$ & $\mathbf{P}$ \\
\hline Age [year] & $58 \pm 11$ & $55 \pm 12$ & 0.74 \\
Gender: male/female & $21 / 17$ & $22 / 14$ & 0.64 \\
Diabetes mellitus & $11(29 \%)$ & $12(33 \%)$ & 0.68 \\
NYHA functional class & $2.3 \pm 0.4$ & $2.2 \pm 0.4$ & 0.81 \\
Heart rate [bpm] & $81 \pm 13$ & $79 \pm 14$ & 0.68 \\
Systolic blood pressure [mm Hg] & $118 \pm 16$ & $114 \pm 14$ & 0.38 \\
Diastolic blood pressure [mm Hg] & $76 \pm 14$ & $74 \pm 12$ & 0.64 \\
QRS duration [ms] & $126 \pm 32$ & $114 \pm 23$ & 0.48 \\
Medications: & $34(90 \%)$ & & 0.74 \\
ACEl or ARB use & $28(74 \%)$ & $33(92 \%)$ & 0.48 \\
Diuretic use & & $29(81 \%)$ & 0.42 \\
Echocardiography: & $218 \pm 52$ & & 0.68 \\
LV end-diastolic volume [mL] & $143 \pm 40$ & $204 \pm 44$ & 0.90 \\
LV end-systolic volume $[\mathrm{mL}]$ & $31 \pm 6$ & $133 \pm 33$ & 0.21 \\
LV ejection fraction [\%] & $67 \pm 5$ & $32 \pm 4$ & 0.64 \\
LV dyssynchrony [ms] & $53 \pm 18$ & $51 \pm 17$ & 0.16 \\
LV-RV dyssynchrony [ms] & $1614 \pm 685$ & $1686 \pm 730$ & \\
NT-proBNP [pg/mL] & & & \\
\hline
\end{tabular}

Results are presented as mean \pm standard deviation; NYHA — New York Heart Association functional class; ACEI — angiotensin converting enzyme inhibitor; ARB - angiotensin receptor blocker; LV — left ventricular; LV dyssynchrony — intraventricular delay; LV-RV dyssynchrony — interventricular delay; NT-proBNP — N-terminal-pro-B-type natriuretic peptide

at the end of the sixth month $(\triangle \mathrm{LVEF}, 7 \pm 2 \%$ to $5 \pm 3 \%, \mathrm{p}=0.02$, Table 3$)$. In addition, both LVEDV and LVESV significantly decreased from baseline to 6 months in the two groups (Table 2). When compared with baseline values change in LVEDV at 6 months ( $\triangle$ LVEDV, $50 \pm 15$ to $40 \pm 17 \mathrm{~mL}$, $\mathrm{p}=0.03)$ was higher in carvedilol group but change in LVESV ( $\Delta$ LVESV, $25 \pm 15$ to $23 \pm 9 \mathrm{~mL}$, $\mathrm{p}=0.28$ ) was similar in the two groups (Table 3 ). LV reverse remodeling (LVESV decrease $>10 \%$ ) was observed in $24(63 \%)$ patients in carvedilol group and 25 (69\%) patients in metoprolol group.

\section{Intra- and interventricular dyssynchrony}

During the 6-month follow-up intraventricular delay decreased from $67 \pm 6$ to $58 \pm 10 \mathrm{~ms}(\mathrm{p}<0.001)$ in carvedilol group and $69 \pm 7$ to $60 \pm 6 \mathrm{~ms}$ $(\mathrm{p}<0.001)$ in metoprolol group (Table 2). Improvement in intraventricular delay at 6 months was similar in the two groups ( $\Delta$ intraventricular delay, $9 \pm 7$ to $9 \pm 6 \mathrm{~ms}, \mathrm{p}=0.91$, Table 3 ). However, improvement in interventricular delay at 6 months was higher in carvedilol group ( $\Delta$ interventricular delay, $11 \pm 8$ to $6 \pm 7 \mathrm{~ms}, \mathrm{p}=0.03$, Table 3 ). At the end of 6 months, there were no differences regarding intra- and interventricular delay between the two groups (Fig. 2). Intra- and inter-observer variabilities for intra- and interventricular dyssynchrony were $3.4 \%, 6.2 \%$ and $3.0 \%, 6.8 \%$, respectively.

\section{NT-proBNP levels}

NT-proBNP values decreased significantly both in carvedilol $(1614 \pm 685$ to $654 \pm 488 \mathrm{pg} / \mathrm{mL})$ and metoprolol (1686 \pm 730 to $583 \pm 396 \mathrm{pg} / \mathrm{mL})$ groups at 6 months $(\mathrm{p}<0.001$ for both). However, decrease in plasma NT-proBNP level was similar in the two groups $(\Delta$ NT-proBNP, $960 \pm 38$ to $1103 \pm$ $\pm 470, \mathrm{p}=0.09)$. Also, the alteration in plasma NT-proBNP levels was positively correlated with a decreas in intraventricular dyssynchrony (Fig. 3).

\section{Discussion}

This study demonstrates that $\beta$-blockage with either carvedilol or metoprolol succinate in addition to standard medical therapy improves $\mathrm{LV}$ dyssynchrony, LVEF, LVESV and LVEDV. Decrease in NT-proBNP level accompanies improvement in LV function and dyssynchrony. Although carvedilol group showed higher decrease in LVEDV, and increase in LVEF, both drugs significantly improved LV dyssynchrony.

Approximately one third of the patients with $\mathrm{HF}$ demonstrate intraventricular conduction abnormalities [12]. Cardiac resynchronization therapy (CRT) improves symptoms and prolongs life in patients with refractory systolic HF and wide QRS complex (> $120 \mathrm{~ms}$ ) on ECG [13]. In past years it was believed that wide QRS on ECG was the most 
Table 2. Comparison of the effects of carvedilol and metoprolol succinate over intra- and interventricular dyssynchronies, ventricular reverse remodeling and hemodynamics in the first and sixth months.

\begin{tabular}{|c|c|c|c|}
\hline & Carvedilol $(n=38)$ & Metoprolol succinate $(n=36)$ & $\mathbf{P}$ \\
\hline \multicolumn{4}{|c|}{ NYHA functional class: } \\
\hline Baseline & $2.3 \pm 0.4$ & $2.2 \pm 0.4$ & 0.81 \\
\hline $1^{\text {st }}$ month & $1.8 \pm 0.5^{*}$ & $1.8 \pm 0.4^{*}$ & 0.73 \\
\hline $6^{\text {th }}$ month & $1.6 \pm 0.5^{*}$ & $1.7 \pm 0.3^{*}$ & 0.42 \\
\hline \multicolumn{4}{|c|}{ Systolic blood pressure [mm Hg] } \\
\hline Baseline & $118 \pm 16$ & $114 \pm 14$ & 0.38 \\
\hline $1^{\text {st }}$ month & $113 \pm 14$ & $109 \pm 18^{*}$ & 0.57 \\
\hline $6^{\text {th }}$ month & $107 \pm 15^{*}$ & $103 \pm 13^{*}$ & 0.74 \\
\hline \multicolumn{4}{|c|}{ Diastolic blood pressure [mm Hg] } \\
\hline Baseline & $76 \pm 14$ & $74 \pm 12$ & 0.64 \\
\hline $1^{\text {st }}$ month & $74 \pm 10$ & $71 \pm 15$ & 0.41 \\
\hline $6^{\text {th }}$ month & $71 \pm 9 *$ & $70 \pm 13$ & 0.77 \\
\hline \multicolumn{4}{|c|}{ Heart rate $[\mathrm{bpm}]$} \\
\hline Baseline & $81 \pm 13$ & $79 \pm 14$ & 0.68 \\
\hline $1^{\text {st }}$ month & $75 \pm 14^{*}$ & $73 \pm 11^{*}$ & 0.77 \\
\hline $6^{\text {th }}$ month & $72 \pm 10^{*}$ & $70 \pm 9 *$ & 0.92 \\
\hline \multicolumn{4}{|c|}{ LV ejection fraction [\%] } \\
\hline Baseline & $31 \pm 6$ & $32 \pm 4$ & 0.90 \\
\hline $1^{\text {st }}$ month & $35 \pm 7^{*}$ & $34 \pm 5^{*}$ & 0.79 \\
\hline $6^{\text {th }}$ month & $38 \pm 6^{* \#}$ & $37 \pm 6^{* \#}$ & 0.52 \\
\hline \multicolumn{4}{|c|}{ LV end-diastolic volume [mL] } \\
\hline Baseline & $218 \pm 51$ & $204 \pm 44$ & 0.42 \\
\hline $1^{\text {st }}$ month & $183 \pm 47^{*}$ & $180 \pm 37^{*}$ & 0.67 \\
\hline $6^{\text {th }}$ month & $168 \pm 39 * \#$ & $164 \pm 34^{* \#}$ & 0.84 \\
\hline \multicolumn{4}{|c|}{ LV end-systolic volume [mL] } \\
\hline Baseline & $143 \pm 36$ & $133 \pm 33$ & 0.68 \\
\hline $1^{\text {st }}$ month & $131 \pm 35^{*}$ & $117 \pm 20^{*}$ & 0.55 \\
\hline $6^{\text {th }}$ month & $118 \pm 29 * \#$ & $110 \pm 25^{* \#}$ & 0.73 \\
\hline \multicolumn{4}{|c|}{ LV dyssynchrony [ms] } \\
\hline Baseline & $67 \pm 6$ & $69 \pm 7$ & 0.21 \\
\hline $1^{\text {st }}$ month & $62 \pm 8^{*}$ & $64 \pm 5$ & 0.45 \\
\hline $6^{\text {th }}$ month & $58 \pm 10^{* \#}$ & $60 \pm 6^{*}$ & 0.28 \\
\hline \multicolumn{4}{|c|}{ LV-RV dyssynchrony [ms] } \\
\hline Baseline & $53 \pm 18$ & $51 \pm 17$ & 0.64 \\
\hline $1^{\text {st }}$ month & $47 \pm 16^{*}$ & $48 \pm 14$ & 0.70 \\
\hline $6^{\text {th }}$ month & $42 \pm 15^{* \#}$ & $45 \pm 15^{* \#}$ & 0.88 \\
\hline \multicolumn{4}{|c|}{ NT-proBNP [pg/L] } \\
\hline Baseline & $1614 \pm 685$ & $1686 \pm 730$ & 0.16 \\
\hline $1^{\text {st }}$ month & $958 \pm 621 *$ & $1132 \pm 776^{*}$ & 0.32 \\
\hline $6^{\text {th }}$ month & $654 \pm 488^{* \#}$ & $583 \pm 396^{* \#}$ & 0.43 \\
\hline
\end{tabular}

Results are presented as mean \pm standard deviation; abbreviations as in Table $1 ;{ }^{*} p<0.05$ between baseline and $1^{\text {st }}$ month, $6^{\text {th }}$ month; ${ }^{\#} \mathrm{p}<0.05$ between $1^{\text {st }}$ month and $6^{\text {th }}$ month

Table 3. Change in selected variables during 6 months of treatment with either carvedilol or metoprolol succinate.

\begin{tabular}{lccc}
\hline & Carvedilol $(\mathbf{n}=\mathbf{3 8})$ & Metoprolol succinate $(\mathbf{n}=\mathbf{3 6})$ & $\mathbf{P}$ \\
\hline$\Delta$ Ejection fraction [\%] & $7 \pm 2$ & $5 \pm 3$ & 0.02 \\
$\Delta$ LV end-diastolic volume $[\mathrm{mL}]$ & $50 \pm 15$ & $40 \pm 17$ & 0.03 \\
$\Delta$ LV end-systolic volume $[\mathrm{mL}]$ & $25 \pm 15$ & $23 \pm 9$ & 0.28 \\
$\Delta$ Intraventricular delay $[\mathrm{ms}]$ & $9 \pm 7$ & $9 \pm 6$ & 0.91 \\
$\Delta$ Interventricular delay $[\mathrm{ms}]$ & $11 \pm 8$ & $6 \pm 7$ & 0.03 \\
$\Delta$ NT-proBNP level $[\mathrm{pg} / \mathrm{mL}]$ & $960 \pm 38$ & $1103 \pm 470$ & 0.09 \\
\hline
\end{tabular}

Results are presented as mean \pm standard deviation; $\Delta$ Change in selected variables during 6-month treatment; abbreviations as in Table 1 


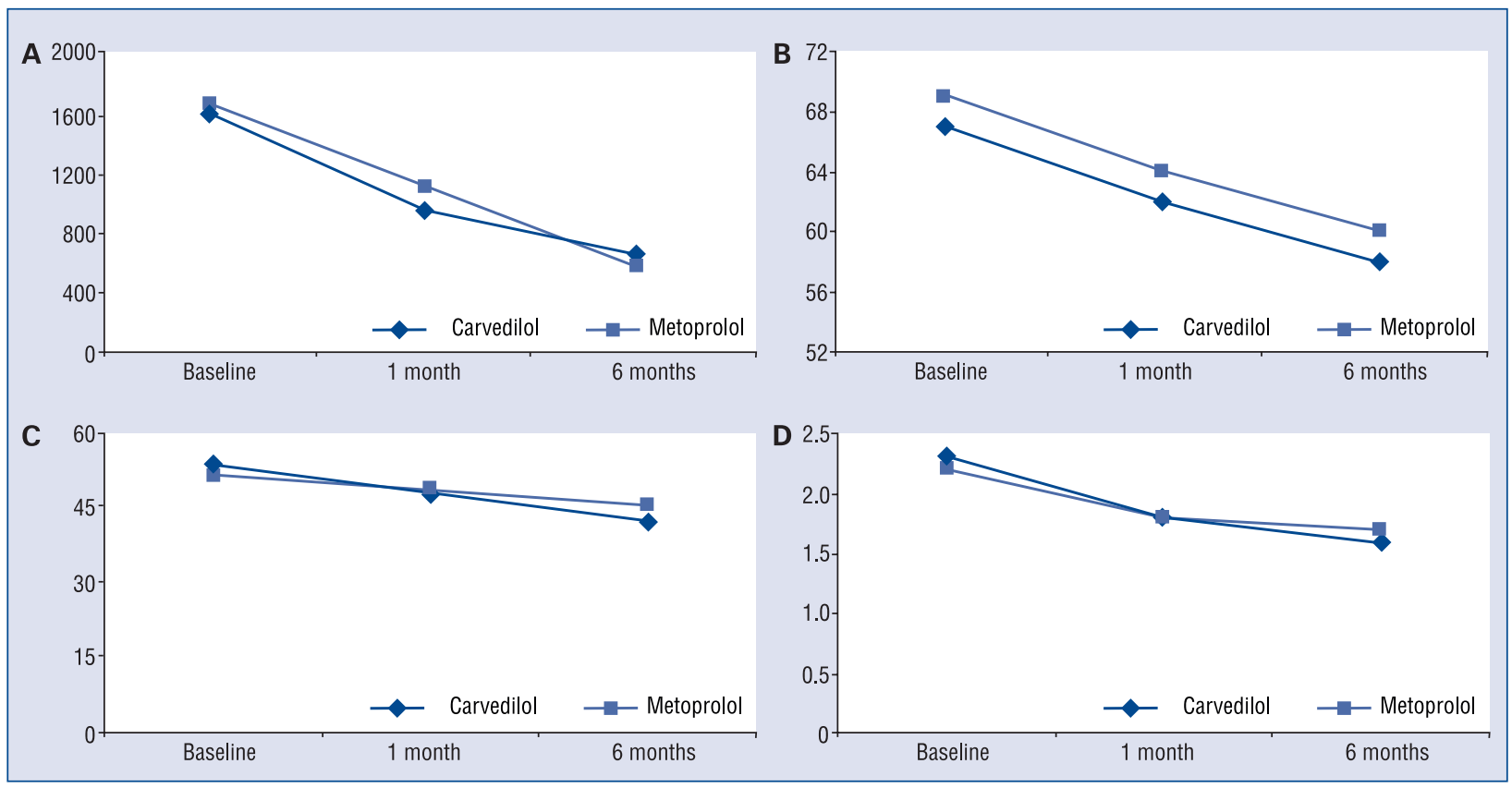

Figure 2. Representative graphics demonstrating the effects of beta-blocker therapy: Changes in N-terminal-pro-B-type natriuretic peptide (NT-proBNP) values (A), intraventricular delay (B), interventricular delay (C), and New York Heart Association (NYHA) classes (D).

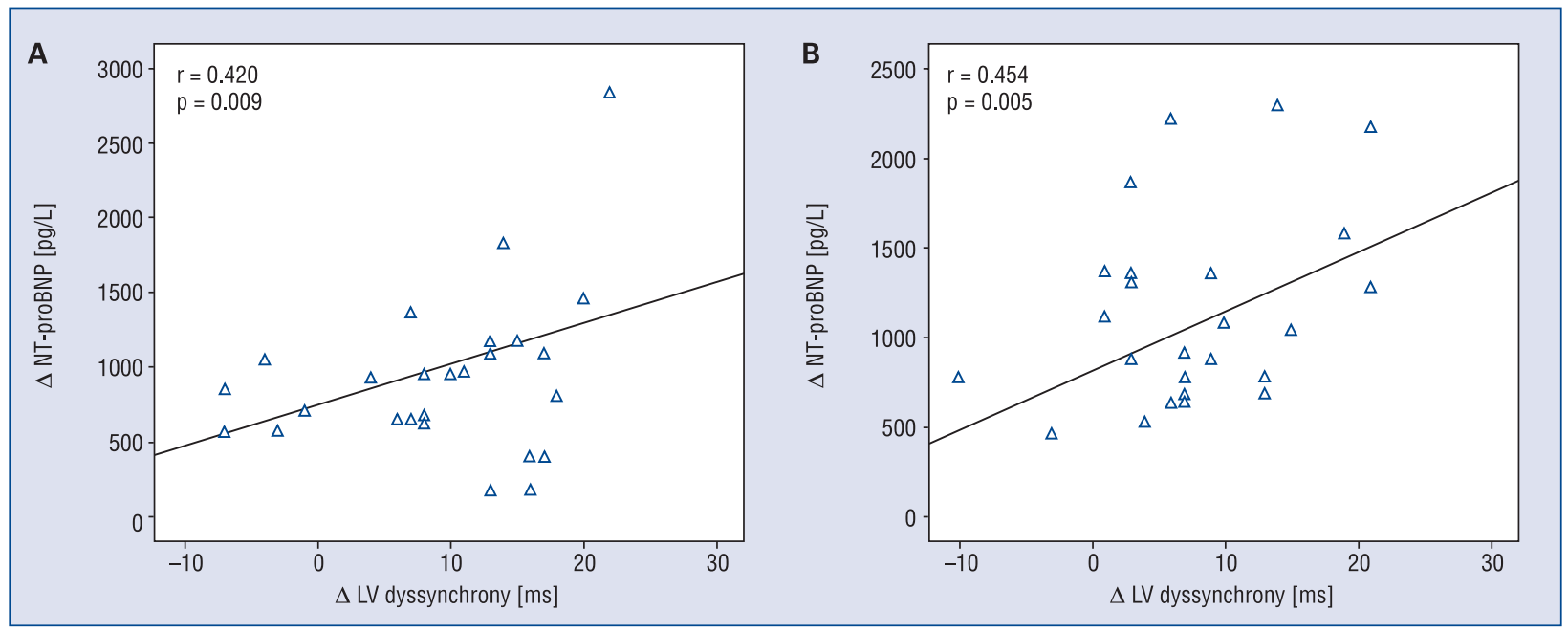

Figure 3. Correlations between changes in plasma N-terminal-pro-B-type natriuretic peptide (NT-proBNP) levels and left ventricular (LV) intraventricular dyssynchrony $[\mathrm{ms}]$ in carvedilol group (A) and in metoprolol group (B).

powerful indicator of intraventricular dyssynchrony. More recently, it has been pointed out that even patients with slightly prolonged or normal QRS may exhibit dyssynchrony [3].

In mid 1990 s, several studies showed that simultaneous electrical stimulation of both ventricles may improve cardiac function in HF patients with intrinsic conduction delay $[14,15]$. In following years, CRT in addition to medical treatment was shown to improve dyssynchrony, induce $L V$ reverse remodeling and reduce mortality in patients with systolic HF [16-18].

Beta-blockers were first introduced for HF treatment in 1975 by Waagstein [19]. Contemporary $\mathrm{HF}$ treatment guidelines recommend addition of carvedilol, metoprolol XL or bisoprolol to conven- 
tional therapy for treatment of systolic HF, based on the results of randomized studies [4-6, 20, 21]. Carvedilol and metoprolol succinate are the most widely used $\beta$-blockers in HF management worldwide. Carvedilol functions by blocking $\beta 1$-, $\beta 2$-, and $\alpha 1$-adrenoreceptor, whereas metoprolol blockes $\beta 1$ adrenoreceptors [22]. Although isolated studies demonstrated a reduction in mechanical dyssynchrony with carvedilol in systolic HF patients $[8,23]$, there is a lack of data comparing its therapeutic effects on mechanical dyssynchrony with metoprolol in IDC patients.

Progressive ventricular dilatation existing in patients with IDC usually accompanies intraventricular dyssynchrony. Optimal medical treatment with $\beta$-blockers have been shown to induce reverse remodeling which may eventually lead to improvement in LV mechanical dyssynchrony [24]. The mechanism of these benefits is likely multifactorial and related to negative chronotropy, which reduces myocardial oxygen consumption, reduction of the impact of high intramyocardial norepinephrine levels [25]. In reversing LV dilatation $\beta$-blockers appear to be superior to the other classes of drugs, with a stronger correlation between dose and effect [26, 27]. Several trials have been conducted to find out the most efficient $\beta$-blocker in improving LV function and reducing LVEDV and LVESV [28]. Ruwald et al. [29] reported less HF hospitalizations or deaths in systolic HF patients taking carvedilol than metoprolol, in particular patients with CRT and left bundle branch block, implying a novel, synergistic effect with carvedilol. However, a meta-analysis written by Chatterjee et al. [30] suggested that the benefits of $\beta$-blockers in systolic HF seem to be mainly due to a class effect, as no statistical evidence from current trials supports the superiority of any single agent over the others.

In our study, although decrease in LVEDV achieved with carvedilol was higher than in the metoprolol group, both drugs led to reverse remodeling and improvement in LV dyssynchrony achieved with these two drugs were similar. We consider that a longer follow up period may be needed to observe the beneficial effects of LV reverse remodeling on intraventricular dyssynchrony. However, carvedilol was superior to metoprolol in restoring interventricular dyssynchrony in our study. Our results clearly confirm the beneficial effects of either $\beta$-blocker on mechanical dyssynchrony at 6 -month follow-up. However, none of these drugs were superior to another in improving intraventricular dyssynchrony. The mechanisms involved in the LV resynchronization with carvedilol or metoprolol succinate are not clearly elucidated. Although carvedilol and metoprolol succinate have different mechanisms of action on $\beta$-receptors, the induction of $L V$ reverse remodeling obtained with both drugs may play a role in the improvement of LV dyssynchrony.

\section{Limitations of the study}

Most of the limitations are inherent to the relatively small sample size and modest follow-up period. Other more advanced echocardiographic techniques like speckle-tracking may be more robust than pulsed-wave TDI in assessing mechanical dyssynchrony. In addition, this study did not use cardiac magnetic resonance to rule out other causes of HF. Lastly, the addition of a placebo group would be helpful in interpreting the results more accurately.

\section{Conclusions}

Both carvedilol and metoprolol improved intraventricular dyssynchrony and induced LV reverse remodeling at 6 months in patients with IDC. The extent of reverse remodeling and reduction in interventricular dyssynchrony achieved with carvedilol was, however, significantly increased when compared to metoprolol.

\section{Conflict of interest: none declared}

\section{References}

1. Farwell D, Patel NR, Hall A et al. How many people with heart failure are appropriate for biventricular resynchronization? Eur Heart J, 2000; 21: 1246-1250.

2. Cazeau S, Leclercq C, Lavergne T et al. Multisite Stimulation in Cardiomyopathies (MUSTIC) Study Investigators. Effects of multisite biventricular pacing in patients with heart failure and intraventricular conduction delay. N Engl J Med, 2001; 344: 873-880.

3. Yu CM, Lin H, Zhang Q et al. High prevalence of left ventricular systolic and diastolic dyssynchrony in patients with congestive heart failure and normal QRS duration. Heart, 2003; 89: 54-60.

4. MERIT-HF Study Group. Effect of metoprolol succinate CR/XL in chronic heart failure: Metoprolol succinate CR/XL Randomised Intervention Trial in Congestive Heart Failure (MERIT-HF). Lancet, 1999; 353: 2001-2007.

5. Poole-Wilson PA, Swedberg K, Cleland JG et al. Comparison of carvedilol and metoprolol on clinical outcomes in patients with chronic heart failure in the Carvedilol or Metoprolol European Trial. Lancet, 2003; 362: 7-13.

6. CIBIS-II Investigators and Committees. The Cardiac Insufficiency Bisoprolol Study II (CIBIS-II): A randomized trial. Lancet, 1999; 353: 9-13.

7. Kamei M, Morita S, Hayashi Y et al. Carvedilol versus Metoprolol for the prevention of atrial fibrillation after off-pump coronary bypass surgery: Rationale and design of the Carvedilol or Meto- 
prolol Post-Revascularization Atrial Fibrillation Controlled Trial (COMPACT). Cardiovasc Drugs Ther, 2006; 20: 219-227.

8. Takemoto Y, Hozumi T, Sugioka K et al. Beta-blocker therapy induces ventricular resynchronization in dilated cardiomyopathy with narrow QRS. J Am Coll Cardiol, 2007; 49: 778-783.

9. Schiller NB, Shah PM, Crawford M et al. Recommendations for quantitation of the left ventricle by two-dimensional echocardiography. American Society of Echocardiography Committee on Standards, Subcommittee on Quantitation of Two-Dimensional Echocardiograms. J Am Soc Echocardiogr, 1989; 2: 358-367.

10. Penicka M, Bartunek J, De Bruyne B et al. Improvement of left ventricular function after cardiac resynchronization therapy is predicted by tissue Doppler imaging echocardiography. Circulation, 2004; 109: 978-983.

11. Bazett HC. An analysis of the time-relations of the electrocardiagram. Heart, 1920; 7: 353-370.

12. Ghio S, Constantin C, Klersy C et al. Interventricular and intraventricular dyssynchrony are common in heart failure patients, regardless of QRS duration. Eur Heart J, 2004; 25: 571-578.

13. Abraham WT, Fisher WG, Smith AL et al. Cardiac resynchronization in chronic heart failure. N Engl J Med, 2002; 346: 1845-1853.

14. Foster AH, Gold MR, McLaughlin JS. Acute hemodynamic effects of atrio-biventricular pacing in humans. Ann Thorac Surg, 1995; 59: 294-300.

15. Kass DA, Chen $\mathrm{CH}$, Curry $\mathrm{C}$ et al. Improved left ventricular mechanics from acute VDD pacing in patients with dilated cardiomyopathy and ventricular conduction delay. Circulation, 1999; 99: 1567-1573.

16. Bristow MR, Saxon LA, Boehmer J et al. Cardiac-resynchronization therapy with or without an implantable defibrillator in advanced chronic heart failure. N Engl J Med, 2004; 350: 2140-2150.

17. Cleland JG, Daubert JC, Erdmann E et al. The effect of cardiac resynchronization on morbidity and mortality in heart failure. N Engl J Med, 2005; 352: 1539-1549.

18. Vatankulu MA, Goktekin O, Kaya MG et al. Effect of long-term resynchronization therapy on left ventricular remodeling in pacemaker patients upgraded to biventricular devices. Am J Cardiol, 2009; 103: 1280-1284.
19. Waagstein F, Hjalmarson $\AA$, Varnaukas E et al. Effect of chronic beta-adrenergic receptor blockade in congestive cardiomyopathy. Br Heart J, 1975; 37: 1022-1036.

20. Dobre D, van Veldhuisen DJ, Mordenti G et al. Tolerability and dose-related effects of nebivolol in elderly patients with heart failure: Data from the Study of the Effects of Nebivolol Intervention on Outcomes and Rehospitalisation in Seniors with Heart Failure (SENIORS) trial. Am Heart J, 2007; 154: 109-115.

21. Udelson JE. Ventricular remodeling in heart failure and the effect of beta-blockade. Am J Cardiol, 2004; 93: 43B-48B.

22. Beattie K, Phadke G, Novakovic J. Carvedilol. Profiles Drug Subst Excip Relat Methodol, 2013; 38: 113-157.

23. Castro PF, Mc-Nab P, Quintana JC et al. Effects of carvedilol upon intra- and interventricular synchrony in patients with chronic heart failure. Am J Cardiol, 2005; 96: 267-269.

24. Doughty RN, Whalley GA, Walsh H et al. Effects of carvedilol on left ventricular remodeling after acute myocardial infarction: The CAPRICORN ECHO study. Circulation, 2004; 109: 201-206 .

25. Eapen Z, Rogers JG. Strategies to attenuate pathological remodeling in heart failure. Curr Opin Cardiol, 2009; 24: 223-229.

26. Frigerio M, Roubina E. Drugs for left ventricular remodeling in heart failure. Am J Cardiol, 2005; 96 (12A): 10L-18L.

27. Sarli B, Topsakal R, Kaya EG et al. Tenascin-C as predictor of left ventricular remodeling and mortality in patients with dilated cardiomyopathy. J Investig Med, 2013; 61: 728-732.

28. Poole-Wilson PA, Swedberg K, Cleland JG et al. Comparison of carvedilol and metoprolol on clinical outcomes in patients with chronic heart failure in the Carvedilol Or Metoprolol European Trial (COMET): Randomised controlled trial. Lancet, 2003; 362: 7-13.

29. Ruwald MH, Ruwald AC, Jons C et al. Effect of metoprolol versus carvedilol on outcomes in MADIT-CRT (multicenter automatic defibrillator implantation trial with cardiac resynchronization therapy). J Am Coll Cardiol, 2013; 61: 1518-1526.

30. Chatterjee S, Biondi-Zoccai G, Abbate A et al. Benefits of beta-blockers in patients with heart failure and reduced ejection fraction: Network meta-analysis. BMJ, 2013; 346: doi: 10.1136/ /bmj.f55. 